\title{
LE LEXIQUE SOMATIQUE DANS L'ARGOT ET LE LANGAGE FAMILIER BULGARES
}

\author{
Gueorgui ARMIANOV \\ INALCO, Université Paris Sorbonne Cité, CREE
}

\begin{abstract}
En): The article analyses the Bulgarian slang vocabulary relating to parts of the human body by comparing it to standard language and colloquial speech and outlining its historical development and current state. Two parallel processes are interpreted in the study: the use of names and expressions of everyday objects to denote parts of the human body and, at the same time, the use of standard, dialectal or colloquial somatic terms to designate physical characteristics or typical personality traits of people. The main semantic processes for creating slang terms, such as metaphor, metonymy, synecdoche, enantiosemy, and semantic derivation, are also discussed. Particular attention is paid to the process of progressive lexical "transfusion" over the last decades of elements of young people corporate slang to colloquial speech and to new urbanolects, as well as to their increasingly larger use in the field literature and the arts, in media and political discourse. The examples used are taken from the Bulgarian press, radio and television broadcasts, internet forums and casual everyday conversations.
\end{abstract}

Keywords (En): standard language; colloquial speech; vernacular; sociolects; slang

Mots-clés (Fr) : standard ; langage familier ; langage populaire ; sociolectes ; argot

DOI : $10.32725 /$ eer.2021.003

\section{Introduction}

En linguistique bulgare, en particulier en dialectologie, il existe de nombreux ouvrages explorant le vocabulaire relatif à la santé et la maladie, la vie et la mort, le travail, la maison, l'amour, le mariage et la vie de famille (VITANOVA, 2015 : 427430 ; VitANOVA, 2016 : 279-307). Or, comme il a été déjà indiqué dans d'autres ouvrages, on n'y parle guère des parties du corps humain et des relations sexuelles (ARMIANOV, 2019: 19-30; ARMIANOV, 2020). Les seules exceptions sont les travaux sur les dialectes régionaux bulgares et les œuvres de folklore, créés sur la base des régiolectes, qui ont toujours été considérés comme faisant partie des recherches sur l'esprit et le caractère nationaux (EMILIYANOVA, $2016: 307-319$; MinAÏLOVA, 2017 : 146-153). Cette situation trouve son explication logique dans la société patriarcale traditionnelle, comme celle de la Bulgarie à la fin du $19^{\mathrm{e}}$ siècle et dans une large mesure même jusqu'à la seconde moitié du $20^{\mathrm{e}}$ siècle, où il était inhabituel de parler ouvertement des parties du corps humain. Toutefois, dans la littérature classique et le folklore, nous pouvons trouver des textes qui évoquent l'apparence physique des personnages, parfois de manière impartiale, parfois avec amour ou humour. Des expressions telles que : snagá topólal, litt. 'stature (comme) un peuplier', oči čeréši 'yeux de cerises', zăbi bíserni 'dents des perles' sont restées dans la langue depuis cette période et, comme elles évoquent des héroïnes de façon

\footnotetext{
${ }^{1}$ En bulgare, les mots ne sont pratiquement jamais accentués, mais ici, pour des raisons de commodité et de meilleure compréhension, l'accent est bien indiqué.
} 
positive et poétique, elles créent une sorte de modèle physique traditionnel de la belle femme. Dans le langage familier, il existe également des comparaisons telles que : rătsé (kato) lopáti 'mains (comme) des pelles', rătsé (kato) čúkove 'mains (comme) des marteaux', ainsi que des termes explicitement moqueurs comme tărboúkh (dial.) et škembé (pers. par le turc işkembe 'tripe') pour le ventre, čétina, 'poils de sanglier' pour les cheveux.

Le corpus analysé est créé sur la base de plusieurs publications consacrées à l'argot bulgare du début du $20^{\mathrm{e}}$ siècle et des premières décennies du $21^{\mathrm{e}}$ siècle, sur le Dictionnaire de l'argot bulgare (ARMIANOv, 2012), sur des conversations enregistrées par l'auteur, ainsi que sur des matériaux provenant de journaux, de forums d'Internet et du site web Bgjargon.

\section{Quelques remarques critiques}

La lacune signalée est encore plus présente dans le vocabulaire somatique des variétés sociales de la langue bulgare : les argots et les urbanolectes modernes. Les mots désignant les parties intimes du corps humain, qui existent sans doute dans la langue et qui sont très bien connus de ses locuteurs, sont généralement perçus comme un élément de la langue vulgaire, leur utilisation est considérée comme une marque de mauvais goût et de culture langagière limitée et leurs locuteurs sont dénoncés comme des vagabonds. Ainsi, dans son article " L'âme du jeune tarikat »", l'écrivain et critique littéraire Efrem Karanfilov décrit les jeunes utilisant l'argot comme des individus antisociaux, vivant en marge de la société et de la loi, qui veulent gagner ou obtenir quelque chose sans effort, sans payer, par la ruse. (KARANFILOV, 1939 : 229-240) On ne peut pas nier le fait que la naissance au $19^{e}$ siècle de l'argot des étudiants bulgares est liée aux argots secrets des artisans et des malfaiteurs, mais une telle description de ses locuteurs est trop éloignée de la réalité, comme cela a été déjà souligné dans la première étude scientifique consacrée aux sociolectes bulgares (STOJKOV, 1945).

\footnotetext{
2 En bulgare, le mot tarikat est un emprunt turc d'origine arabe et il est utilisé pour désigner une personne rusée, maligne. Au début du $20^{\mathrm{e}}$ siècle, il a également désigné les écoliers, les étudiants qui étaient les principaux locuteurs de l'argot.
}

$$
-30-
$$




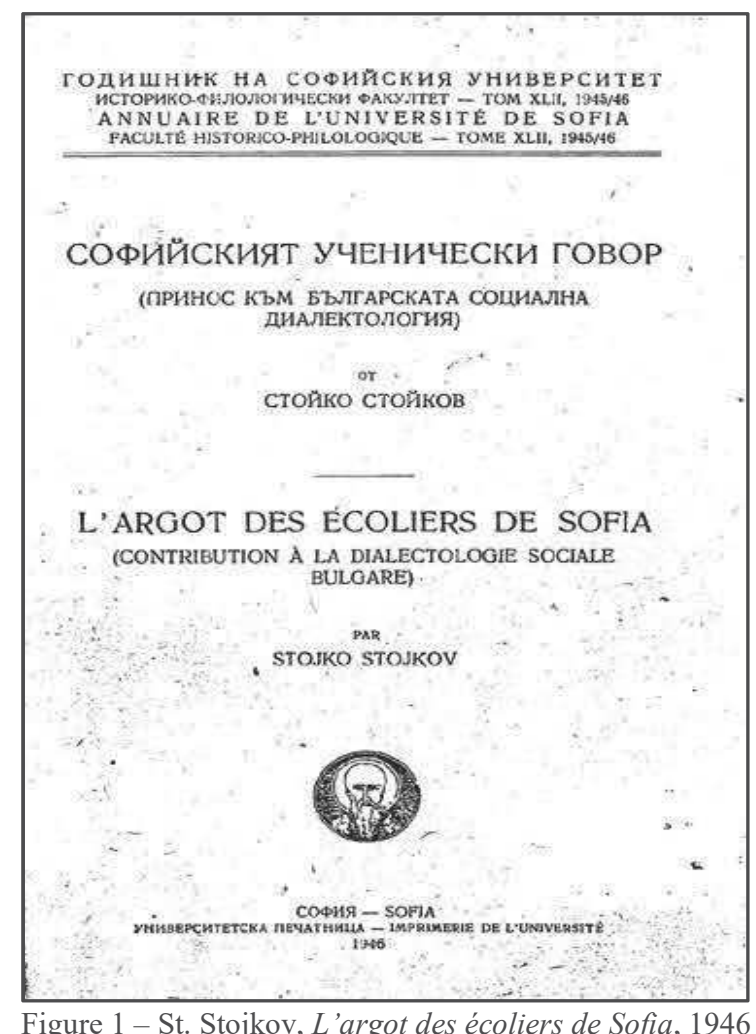

Il faut également ajouter qu'après l'installation du régime communiste en Bulgarie en 1944 et jusqu'aux années 1980, les recherches sur ces variétés non standard de la langue bulgare constituaient plutôt une rareté et elles étaient vues comme une simple perte de temps. Comme résultat d'un tel faux jugement, l'introduction d'argotismes dans les dictionnaires, même accompagnés de marques stylistiques appropriées, était pratiquement exclue. Ainsi, dans les premières éditions du Dictionnaire raisonné bulgare et du Dictionnaire de la langue bulgare jusqu'au vol. 8, le verbe traditionnel et populaire pour l'acte sexuel est absent, ainsi que les mots désignant le pénis et l'organe sexuel féminin. Même de nos jours, trente ans après les changements politiques et idéologiques dans le pays, les controverses restent $^{3}$ et rares sont les exceptions, telles que la nouvelle édition du Dictionnaire raisonné bulgare et l'édition électronique du Dictionnaire de la langue bulgare, dans lesquelles, après moult discussions et sous maintes conditions, seuls quelques-

3 Par exemple: dans la partie bulgare du Dictionnaire français-bulgare/bulgare-français (TCHAOUCHEV 2009) ce verbe est bien présent, mais le terme populaire gắz (2ъ3 en bulgare) 'fesses' est absent, alors qu'il existe dans la définition du mot français cul (TCHAOUCHEV, 2009 : 158). Dans le Nouveau dictionnaire compact français-bulgare/bulgare-français, tous les mots argotiques pour les parties intimes du corps humain sont absents à la fois dans la partie française et la partie bulgare (PONS, 2006). 
uns des mots les plus fréquents des parties intimes du corps humain et des relations sexuelles ont été introduits ${ }^{4}$.

La pudeur linguistique et les restrictions politiques et idéologiques étaient aussi la raison de la distinction assez prononcée en Bulgarie entre l'argot des jeunes et le langage familier. Alors que l'argot était considéré comme une marque de mauvais goût et de culture langagière limitée, le langage familier était généralement ressenti comme une forme libre de la langue standard dans laquelle les argotismes et les somatismes en particulier n'avaient pas leur place.

Pour cette raison, une étude détaillée et impartiale de cette couche lexicale brisera non seulement un tabou centenaire, mais enrichira aussi la perception de certains domaines spécifiques des sociolectes bulgares. On peut noter qu'une petite partie de ce vocabulaire a été déjà évoquée et étudiée dans des recherches contemporaines sur l'argot bulgare et sur sa place dans le tableau des sociolectes européens (ARMIANOV 1995 ; ARMIANOV 2019).

\section{Traits caractéristiques}

Les études récentes sur la langue des jeunes Bulgares mettent en évidence quelques caractéristiques importantes du vocabulaire somatique argotique :

2.1. Le premier trait qui saute aux yeux est sa richesse extraordinaire. Elle s'explique notamment par le fait que le corps est l'un des principaux objets d'attention chez les jeunes et que cette attention est dirigée non seulement vers le sexe opposé, les amis proches ou les rivaux, mais aussi vers les personnes n'appartenant pas au groupe social du locuteur : enseignants, professeurs, parents, personnages célèbres, etc.

Pourtant, la comparaison statistique révèle un paradoxe intéressant: dans le Dictionnaire tarikat-bulgare de P. Voïnikov, le nombre de mots du champ sémantique « corps humain » est très limité - quatre seulement (VOÏNIKOV, 1930 : 66-76), dans l'étude de Stojko Stojkov de 1945 sur l'argot des étudiants de Sofia il y en a vingt-trois (STOJKOV, 1945 : 67-72), alors que dans le Dictionnaire de l'argot bulgare de 2012, leur nombre est proche de 490, y compris plusieurs dizaines de mots et d'expressions pour les personnes en fonction de leurs caractéristiques physiques (ARMIANOV, 2012). Quelle est la raison de ce gigantesque sursaut lexical ? Est-il possible qu'au début du $20^{\mathrm{e}}$ siècle, les jeunes n'aient presque pas parlé des parties du corps humain et que vers sa fin ils aient été «envahis » par 1'euphorie de la création langagière ? La statistique ne nous révèle pas une image réelle. Pour comprendre les raisons de ce paradoxe, il est nécessaire de souligner qu'à la fin du $19^{\mathrm{e}}$ siècle (l'époque de la naissance de l'argot moderne bulgare) ainsi qu'au début du $20^{\mathrm{e}}$ siècle, la société bulgare partageait encore une grande partie des valeurs traditionnelles et patriarcales, comme la pudeur et la timidité langagière, qui jouaient un rôle important dans la vie quotidienne. Au cours du $20^{\mathrm{e}}$ siècle, ces valeurs ont été progressivement abandonnées par la société moderne et, par

\footnotetext{
${ }^{4}$ Dictionnaire de la langue bulgare, https://ibl.bas.bg/rbe/lang/bg/\%D0\%B5\%D0\%B1\%D0\%B0/ (consulté le 22.06.2021).
} 
conséquent, plusieurs mots et expressions bien connus, mais délibérément évités, voire interdits, ont émergé . D'où la richesse lexicale d'aujourd'hui.

2.2. La deuxième caractéristique importante est que le vocabulaire somatique est largement «masculin» et souvent misogyne - une particularité que l'on découvre dans tous les sociolectes juvéniles du monde entier et qui trouve ses racines à la fois dans l'origine traditionnellement masculine de l'argot et probablement dans le fait que les caractéristiques physiques des garçons/des hommes attirent relativement rarement l'attention des filles/des femmes utilisatrices de l'argot. Pour illustrer cette idée, on peut utiliser la figure ci-dessous, indiquant les zones qui fournissent un motif à l'expression verbale en argot (Figure 2) :

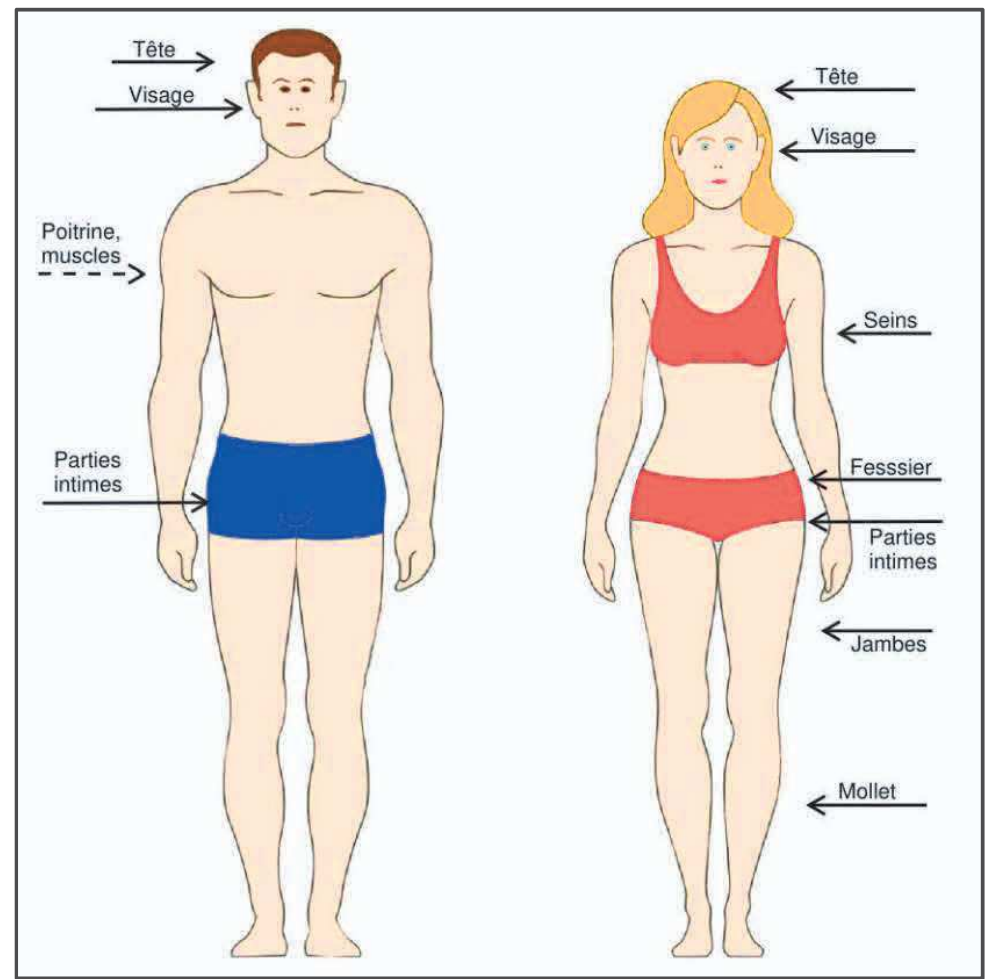

Figure 2 - Zones d'expression verbale

Sur le corps masculin, les zones sont au nombre de quatre et l'une d'elles, les parties intimes et en particulier le pénis, détient une sorte de record pour le nombre de lexèmes - plus de soixante, tandis que la zone de la poitrine et des muscles pectoraux (ligne pointillée /.../) peut être définie comme insignifiante. Sur le corps féminin, il y a sept zones qui sont toutes définies comme importantes, bien qu'à des degrés divers : les plus attrayantes sont les seins, les fesses, les cuisses et les parties intimes. Je trouve indispensable d'indiquer que des correspondances se retrouvent

\footnotetext{
5 À cet égard, il est intéressant de citer le «Dictionnaire des mots abjects censurés. Matériaux non publiés de l'argot bulgare » (ARMIANOV, 1990-1991).
} 
dans les noms de certaines parties du corps chez l'homme et la femme, en particulier la tête et le visage, le nez et les oreilles.

Comme il a été déjà noté, des études récentes sur l'argot en Bulgarie montrent sans équivoque que de plus en plus de filles et de femmes utilisent ce type de vocabulaire, bien qu'avec certaines limites claires : les noms des parties intimes masculines peuvent rarement être repérés dans le discours argotique féminin, mais les autres parties du corps ne posent pas de problème et leur utilisation, le plus souvent dans un contexte moqueur, est un phénomène courant (ARMIANOV, 2020).

2.3. La troisième caractéristique concerne la désignation sémantique, où deux processus parallèles, mais opposés formant deux groupes d'argotismes se dégagent immédiatement :

a) Dans le premier, en conséquence d'une transformation sémantique, des noms et des expressions d'objets quotidiens, tels que fruits, légumes, appareils électroménagers, meubles, etc., sont utilisés pour désigner des parties du corps humain.

b) Dans le deuxième cas, on observe le processus inverse - des somatismes standard, dialectaux ou familiers servent à désigner des caractéristiques physiques ou des traits typiques du caractère des personnes.

La fréquence des termes de deux types est très élevée et son analyse montre la présence significative de mots au sens moqueur ou péjoratif qui témoignent moins d'une antipathie ou de mauvais sentiments, que de la volonté du locuteur de souligner sa propre supériorité ou celle d'un(e) proche.

\section{Somatismes argotiques}

3.1. La tête. Ainsi, à la suite du premier type de transformation sémantique, on trouve près de 50 mots pour désigner la tête par le nom d'un ustensile domestique, comme : báka 'bouteillon', ćáïnik 'bouilloire', čoútoura 'mortier de cuisson', kánče 'timbale, gamelle', kófa 'seau', manéka 'gourde militaire', stómna 'cruche'. Il existe également de nombreux noms de fruits et de légumes, tels que : zélka 'chou', kartóf 'pomme de terre', korávets, păpeš 'melon', loúkovitsa 'bulbe', répička 'radis', tíkva 'citrouille', kratoúna 'calebasse'; ou les noms d'instruments de musique : kitára 'guitare', balaläika 'balalaïka', kemané 'rebec traditionnel', taramboúka 'darbouka' ou ceux qui désignent un objet d'une forme similaire à celle de la tête : glóbous 'globe terrestre', kambanaríya 'clocher', káska 'casque', koubé 'coupole', šamandoúra 'balise marine', yaïtsé litt. 'œuf', kabriolét litt. 'cabriolet' et far 'phare' pour 'tête chauve ou rasée' et bien d'autres 6 . Par extension, certains termes comme les tíkva, păpeš, kratoúna, taramboúka cités ci-dessus sont largement utilisés pour désigner une personne stupide, habituellement un homme, plus rarement une femme.

En comparaison, dans la langue standard, un seul mot, glavá 'tête', est présent dans un certain nombre d'expressions ou de combinaisons idiomatiques populaires telles que glavá na seméïstvo 'chef de famille', dăržáven glavá 'chef de l'état', glavá loúk 'tête d'oignon', glavá na kníga 'chapitre d'un livre'.

\footnotetext{
${ }^{6}$ Les exemples argotiques sont tirés du Dictionnaire de l'argot bulgare (ARMIANOv, 2012).
}

$$
-34-
$$


Dans les dialectes régionaux, il n'existe probablement pas plus d'une dizaine de mots de ce type, dont certains ont été incorporés dans l'argot des étudiants ou dans le langage familier (comme pipón 'melon' pour 'tête' ou kakaláška 'épi de maïs' pour 'pénis'), mais on y trouve aussi d'autres qui, en raison du développement et de la modernisation de la société sont aujourd'hui devenus obsolètes ou presque inconnus, comme : krínče 'petit boisseau' dans la comparaison traditionnelle glavá (kato) krínče, litt. 'une tête comme un boisseau', pour dire 'grosse tête'.

3.2. Le visage. Le visage avec ses éléments nous offre aussi une grande richesse de matière lexicale. Les locuteurs de l'argot l'appellent (d)zoúrla 'museau', ikonostás 'chancel', léguitimátsiya 'identification', moútra 'gueule' (très populaire aujourd'hui dans le langage familier), óbraz 'image', sourát (de l'arabe via le turc surat 'visage'), fasáda 'façade' et fasón du mot français façon, ainsi que le mot composé fiziomoútra qui provient du mot standard fizionómiya 'physionomie' et l'argotisme moútra. Les argotismes pour désigner le nez, qui fascine par sa forme et sa taille, ne sont pas moins nombreux. Ainsi, il est dénommé kámba 'poivron tomate', čoúška 'poivron long', patladžán 'aubergine', mórkov 'carotte'. Il peut être aussi représenté par un grand nombre d'objets et d'instruments mécaniques comme barométăr 'baromètre', máslenitsa et masljónka 'graisseur', otvaráčka 'décapsuleur', ráïber 'fermeture de porte', češmá 'robinet', klyún et čóvka 'bec', tsifka 'sifflet primitif', etc.

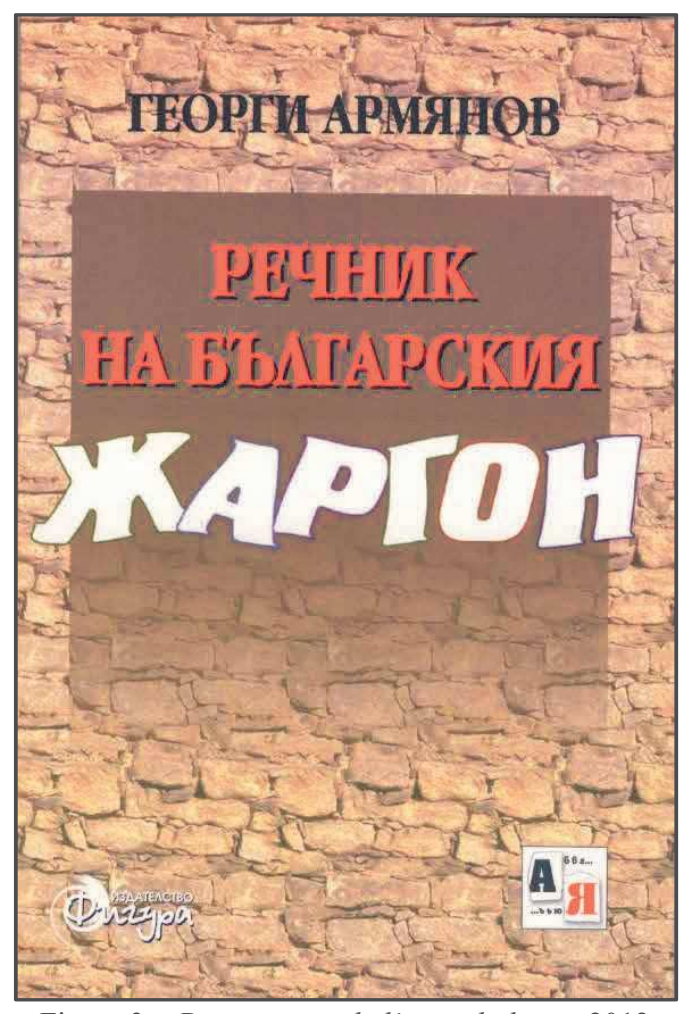

Figure 3 - Dictionnaire de l'argot bulgare, 2012 
Dans l'argot des jeunes Bulgares, la bouche s'appelle balaláïk 'l'instrument musical balalaïka' (juste comme la tête) parce qu'elle tinte, gratte, sonne, plyuválnik 'crachoire', čekmedžé 'tiroir', glifálo 'instrument à bouffer' du verbe argotique glifam 'manger', lapatsálo 'organe qui cliquette', čóvka 'bec'; les yeux son radári 'radars', svetofári 'feux de circulation', skéneri 'scanners', fárove 'phares'; les oreilles sont radári et lokátori 'radars', palačínki 'crêpes', pelengátori 'radiogoniomètres', slušálki 'écouteurs'.

En comparaison, pour le visage le vocabulaire familier est assez pauvre et ne propose que quelques mots provenant soit de la langue standard comme moutsoúna 'gueule' et mórda (du même mot populaire russe), soit de l'argot comme moútra, mais aucun terme pour désigner le nez, les yeux ou les oreilles.

3.3. La poitrine, l'abdomen, les épaules et les parties postérieures. Ensuite, les champs lexico-sémantiques de l'argot des jeunes Bulgares pour les corps masculin et féminin commencent à diverger, à la fois en termes de zones favorites et en termes de richesse. Ainsi, pour la partie supérieure du corps masculin - la poitrine et les muscles pectoraux - on trouve un seul lexème, pérki, litt. 'nageoires', et seulement quelques mots pour les autres parties, comme : bitsi, troncation du mot biceps, plóčki, litt. 'carreaux', pour les muscles bien formés de l'abdomen. On peut ajouter ici l'argotisme garderób 'garde-robes', qui signifie 'homme fort aux épaules larges ${ }^{77}$ et qui aujourd'hui fait plutôt partie du vocabulaire familier qu' argotique.

Le ventre accentue encore les différences entre l'argot «masculin» et " féminin »: on retrouve des mots tels que avtoritét 'autorité', bóiler 'chaudière', meh et méška 'grand sac', otéčestvo 'patrie', samočoúvstvie 'confiance', ainsi que les emprunts dialectaux et familiers tărboúkh et škembé déjà mentionnés. Or, presque tous sont utilisés pour le ventre masculin et pratiquement aucun pour celui de la femme. En revanche, les seins féminins attirent l'attention des garçons et donnent naissance à l'un des groupes lexicaux argotiques les plus riches, comportant plus de vingt mots et expressions comme : avangárd 'avant-garde', amortisióri 'amortisseurs', balkón 'balcon', bómbi 'bombes', boúferi de l'anglais buffer 'tampon de voiture ou de train', bămperi de l'anglais bumper 'pare-chocs', dihátelno oustróïstvo 'appareil respiratoire', dyúli 'coings', érkeri 'fenêtre en saillie, en baie', etažérka 'étagère', kroúši 'poires', motsaréli/motsarélki '(petites) boules de mozzarella', oči 'yeux', planiní 'montagnes', portokáli 'oranges', silikónki 'boules de silicone' et plusieurs autres.

La partie postérieure de la hanche nous fournit aussi un nombre assez impressionnant d'argotismes, mais presque la totalité désigne les fesses masculines : gevrék litt. 'bagel traditionnel', géizer 'geyser', gălăbárnik litt. 'colombier', diferentsiál 'différentiel', priemátel litt. 'récepteur', părdálnik litt. 'organe qui pète', driskálnik et kendzálnik 'organe qui chie', sedálka 'siège', záden dvor 'la cour arrière', tórta 'gâteau', halká 'anneau' et une dizaine d'autres, alors que seuls les deux derniers termes ainsi que le mot standard doúpe ('bulg. dyne 'fesses') et l'euphémisme familier boúkvi, litt. 'lettres', peuvent être employés pour une femme.

\footnotetext{
${ }^{7}$ En argot français, ou selon certains linguistes et lexicographes en langage familier, on découvre une expression dont la signification est assez similaire: armoire à glace "personne de carrure impressionnante' (Le Petit Robert, 1993 : 122).
} 
Il faut ajouter que dans le discours des jeunes, ainsi que de beaucoup d'autres personnes, d'habitude peu cultivées, l'on découvre souvent le mot populaire et vulgaire găz 'cul', mais il reste clairement séparé du vocabulaire argotique et son utilisation n'est que rarement associée à l'argot.

3.4. Les membres supérieurs et inférieurs. Les argotismes pour les jambes sont souvent les mêmes pour les hommes et les femmes, par exemple : amortisïóri 'amortisseurs', džóngali 'jambes longues' emprunt à la langue romani, kápli litt. 'jantes' pour 'pieds nus', krénvirši 'saucisses', makaróni 'pâtes', pergéli litt. 'compas', spitsi litt. 'rayons d'une roue de bicyclette', štéki 'bâtons pour le ski'. Une grande partie des mots cités ici existent uniquement ou sont utilisés majoritairement au pluriel, bien que l'on puisse signaler des exceptions, comme l'expression otáryam/raztyágam pergéla 'litt. 'ouvrir/étirer le compas' pour dire 'marcher vite, à grands pas' où l'argotisme pergél 'compas' est au singulier8.

Il existe également des mots différents, tels que le badžák et bedró 'cuisse' ou baldăr 'mollet; cuisse', utilisés uniquement pour les femmes. Il est intéressant de noter que pour la main ou le bras en argot on trouve des mots qui, selon mes observations, s'appliquent plus aux hommes qu'aux femmes, tels que : băzikálo du verbe băzikam 'farfouiller', gepálo du verbe gépvam (imperf.)/gépya (perf.) 'attraper' et lápa, litt. 'patte' pour désigner la main ou toupálka litt. 'batteur de tapis' pour une main ou un bras fort d'un homme.

3.5. Les parties intimes. Une autre zone, qui attire particulièrement l'attention des jeunes et stimule leur créativité, est la zone intime. Là encore, il y a un nombre remarquable d'argotismes, mais cette fois-ci en relation tout autant avec le corps féminin, qu'avec le corps masculin. Les organes génitaux ressemblent le plus souvent à un objet, un fruit ou un légume. Pour le membre masculin, cela peut être găba 'champignon', krástavitsa 'concombre', salám 'saucisson', krénvirš 'saucisse', kočán 'épi de maïs égrené', tsárevitsa 'épi de maïs', šišárka 'pomme de pin', mais aussi bastoún 'canne', toyága 'bâton', kavál et doudoúk 'flûte traditionnelle', džóistik 'joystick', markoúč 'tuyau', rog 'corne', hoúrka 'quenouille', čep 'nœud d'un arbre'. On découvre aussi des expressions particulièrement figuratives telles que : kavál s moustátsi 'flûte à moustache', kóžen pirón 'clou en cuir', tréti krak 'troisième jambe' et sréden krak 'jambe de milieu' et plus encore. À cette liste peuvent être ajoutés les noms argotiques des testicules, tels que : bižoúta 'bijoux', torbi et disági 'sacs', kartófi 'pommes de terre', makari 'bobines', órehi 'noix', tópki 'boules' et une dizaine d'autres. Pour le vagin les noms dépassent également une vingtaine, certains assez vulgaires. Le Dictionnaire de l'argot bulgare déjà cité nous présente des mots comme : doúda 'mûre', kaïsíya 'abricot', sliva 'prune', káteritsa 'écureuil', kótentse 'chaton', mekitsa 'galette', mída 'moule', míndža du mot romani mindž 'vagin', pizzda 'chatte' mot d'origine proto-slave qui existe pratiquement dans tous les argots slaves, pónička 'beignet',

\footnotetext{
${ }^{8}$ Sur le site d'Internet Bgjargon, on découvre un très grand nombre de mots présentés comme argotiques qui, en réalité, sont dialectaux, familiers, parfois même standard (https://www.bgjargon.com, consulté le 22.06.2021).

${ }^{9}$ On trouve le mot abricot avec la même signification dans l'argot des jeunes Français (Parisien 2019 :

15). Par contre, dans l'argot des jeunes Moskovites, le mot signifie 'testicules' (Elistratov, 1994 : $11)$.
} 
dyukyán, litt. 'braguette', de l'arabe via le turc dükkân 'boutique', méždoumétie 'interjection', etc.

Il est intéressant de dévoiler ici que, dans plusieurs enquêtes que j'ai menées pour la première fois en 1981 auprès d'étudiants bulgares dans quatre grandes villes et que j'ai refaites par la suite en 1992 et 2002, les jeunes garçons interrogés, tout comme les jeunes filles, étaient bien gênés de me fournir des mots argotiques désignant les parties intimes du corps ou ils répétaient souvent des mots standard ou familiers. Évidemment, la présence d'un étranger, plus âgé qu'eux, ainsi que la forme écrite de l'enquête (leurs réponses, bien qu'anonymes, sont restées comme une sorte de preuve écrite) ont joué un rôle majeur dans cette difficulté de s'exprimer. Dans une atmosphère détendue, lorsque j'écoutais leurs conversations comme un parfait inconnu, ils (surtout les garçons) n'ont pas hésité à utiliser les argotismes que j'ai cherché à dénicher avec mes enquêtes.

\section{Procédés sémantiques}

Les principaux procédés de création de termes argotiques peuvent être divisés en deux groupes : le premier, qui est très productif et fournit la grande majorité des somatismes, est illustré essentiellement par la métaphore universelle ou conceptuelle. La transposition métaphorique donne la possibilité aux locuteurs d'argot de faire preuve d'originalité, mais ce n'est pas uniquement un jeu de langage. Il est vrai que ce procédé produit une atmosphère " poétique » inattendue et peut même provoquer le rire, mais son but principal est ailleurs : au moyen de la transposition métaphorique, d'une part, le système lexical argotique s'enrichit et, d'autre part, les mots et les expressions vieillis, pâles, usés par l'emploi sont remplacés et repoussés à la périphérie. Dans ce groupe, même si plus rarement, on rencontre également la dérivation sémantique et la dérivation antonymique, la métonymie et les euphémismes (ARMIANOV 1995 : 101-123). Dans ce groupe, nous pouvons mettre la plupart des mots désignant la tête, le nez, les membres supérieurs et inférieurs, ainsi que les mots et les expressions pour les parties intimes du corps.

Le deuxième groupe de somatismes non-standard est nettement moins productif et, d'après mes observations, les termes relèvent davantage du domaine du langage familier que de l'argot des jeunes. Une autre caractéristique que l'on remarque immédiatement est que la plupart des mots sont créés sur le principe de la synecdoque du type pars pro toto et de l'énantiosémie. Ici, on peut indiquer des mots comme: mózăk, litt. 'cerveau', qui désigne un homme extrêmement intelligent, mais également un homme très stupide, găz et zádnik, litt. 'cul, fesse', qui signifie 'homme stupide, hébété', bedró, litt. 'cuisse', pour une belle fille aux jambes longues, les termes cités ci-dessus tărboúkh et škembé, litt. 'ventre', pour un gros homme qui ne pense qu'à manger', ouhó 'oreille' pour un agent secret, espion, grăb, litt. 'dos', pour une personne forte et influente qui peut éviter les ennuis à quelqu'un, porazyávaštata răká, litt. 'la main qui endommage' pour un dentiste incompétent (jeu de mots inspiré de Old Shatterhand, nom du héros des romans d'aventures de l'écrivain allemand Karl May), tašak et testis, qui viennent respectivement de la langue turque et du latin et qui signifient 'testicule', dans 
l'argot bulgare ont acquis une nouvelle signification - 'blague, moquerie', ainsi que plusieurs mots vulgaires bien connus, désignant le sexe féminin, mais souvent utilisés pour définir une femme ou une fille séduisante.

\section{Conclusion}

L'analyse du vocabulaire somatique non standard bulgare nous montre une approche langagière manifestement masculine et une richesse lexicale énorme. Toutefois, il est indispensable de souligner deux points importants. Premièrement, les filles et les femmes s'approprient davantage d'argot dit «masculin» et aujourd'hui on peut facilement repérer des argotismes dans leur discours quotidien. Deuxièmement, au cours des dernières décennies, on observe un processus de «transfusion » lexicale progressive d'éléments de l'argot corporatif des jeunes vers le langage familier et les nouveaux urbanolectes, ainsi que leur utilisation de plus en plus large dans la littérature et les arts de la scène, dans les médias et même dans le discours politique. Par exemple, le discours du Premier ministre bulgare (20092013) Boïko Borissov était souvent parsemé d'argotismes bien connus qui de plus en plus sont considérés comme appartenant au langage familier et, par conséquent, sont largement tolérés.

De surcroît, certains argotismes et mots familiers ont été inclus dans quelques manuels scolaires récents pour illustrer les diverses variétés linguistiques substandard du bulgare. Il s'agit d'un processus normal de changement dans la perception de l'argot, ainsi que de l'évolution graduelle de la base linguistique de l'argot : d'une base dialectale et populaire à une base originale, nouvellement créée.

\section{BIBLIOGRAPHIE}

ANDREJCHIN Lubomir et al. (2012), Български тълковен речник (4éd.), Sofia, Nauka i izkustvo.

ARMIANOV Gueorgui (1990/1991), Цензурираният нецензурен речник, Свободна книга, n. 2-3, 1990 ; n. 1, 2, 3, 5, 1991, Sofia.

ARMIANOV Gueorgui (1995), Българският жаргон - лексико-семантичен и лексикографски аспект, Sofia, Université « Saint Kliment Ohridski ».

ARMiANOv Gueorgui (2012), Речник на българския жаргон, Sofia, Figura.

ARMIANOV Gueorgui (2019), Le vocabulaire culinaire des variétés substandard bulgares: entre l'Orient et l'Occident, in: BASTIAN Sabine, Felten Uta, GoudAillier Jean-Pierre (éd.), Sprache-Kultur-Geselschaft 21. Cultures et mots de la table, Berlin, Peter Lang.

ARMiANOV Gueorgui (2020), Tout est amour (l'amour et la sexualité dans les variétés non standard de la langue bulgare), in : Acta Universitatis Lodziensis, Folia Litteraria Romanica (à paraître).

BGJARGON 2020, https://www.bgjargon.com.

Cholakova Kristalina, éd. (1977), Речник на българския език, vol. 1-8, Sofia, BAN. 
ELISTRATOV Vladimir (1994), Словарь, московского арго, Moscou, Russkie slovari.

EMILIYANOVA Boryana (2016), Соматизмът „череп“ в българската народна географска терминология и топонимия, Състояние и проблеми на българската ономастика 1 (14), Sofia.

KARANFILOV Efrem (1939), Душата на младежа-тарикат, Философски преглед $X I$ (3), Sofia.

Le Nouveau Petit Robert, (1993) Paris, Dictionnaires Le Robert.

Le Parisien (2019), 03.10.2019.

MiнAїLOVA Boryana (2017), Метафорични народни географски термини, които произлизат от названието на лицето в българския и в румънския език, in : Балканите - език, история, култура, vol. 5, Veliko Turnovo.

PONS (2006), Nouveau dictionnaire compact français-bulgare/bulgare-français, Stuttgart, Ernst Klett Sprachen GmbH ; Sofia, PONS Bulgaria EOOD.

StoJKov Stojko (1946), Софийският ученически говор, in: Годишник на Софийския университет 1945/46, XLII, Sofia.

TCHAOUCHEV Assen (2009), Dictionnaire français-bulgare/bulgare-français, Sofia, Colibri.

VitANOVA Mariyana (2015), Здравето и болестта в лексикалната система на българските диалекти, Journal of Process Management - New Technologies (8/1), Vranje, Serbia.

VITANOV Mariyana (2016), Концептьт труд/работа в българската езикова картина на света, in : Leksykon aksjologiczny Stowian i ich sasiadów, T. 3 PRACA, Lublin, Wydawnictwo UMCS.

VoÏNIKOV Petko (1930), Тарикатско-български речник, Родна реч 4, Sofia. 